\title{
Slowly Developing Progressive Familial Intrahepatic Cholestasis Type 2 (PFIC2) in an Adolescent with Cholelithiasis, Gilbert's Syndrome and Chronic Calcifying Pancreatitis
}

\author{
M. Hartleb ${ }^{*}, 1$, M. Jirsa ${ }^{2}$, M. Zindr ${ }^{2}$, J. Pawłowska ${ }^{3}$, I. Jankowska ${ }^{3}$ and J. Cielecka-Kuszyk ${ }^{3}$ \\ ${ }^{I}$ Department of Gastroenterology, Medical University of Silesia, Katowice, Poland \\ ${ }^{2}$ Laboratory of Experimental Hepatology, Institute of Clinical and Experimental Medicine, Praha, Czech Republic \\ ${ }^{3}$ Department of Gastroenterology, Hepatology and Immunology, Children's Memorial Health Institute, Warsaw, Poland
}

\begin{abstract}
Progressive familial intrahepatic cholestasis type 2 (PFIC2) is a rapidly developing hepatic disease that leads to early childhood cirrhosis and liver failure. We present a diagnostically challenging case of a 20 -year-old male with 8 years history of recurrent icteric episodes and constantly normal serum levels of GGT. Genetic study disclosed two novel mutations in gene $A B C B 11$ and liver histopathology provided evidence of intrahepatic cholestasis with slowly progressing fibrosis. Concurrent diseases were cholelithiasis and chronic calcifying pancreatitis.
\end{abstract}

\section{INTRODUCTION}

Progressive familial intrahepatic cholestasis type 2 (PFIC2), caused by mutations in $A B C B 11$, is a rapidly progressing disease leading inevitably to cirrhosis, liver failure, and occasionally to cholangiocarcinoma or hepatocellular carcinoma in early childhood [1, 2]. Patients with PFIC2 usually require liver transplantation before age 10 .

Recurrent self-limiting cholestatic jaundice suggests a "benign mutation" that does not result in significant injury to the liver (benign recurrent intrahepatic cholestasis; BRIC). At present, PFIC2 and BRIC2 are not considered clinically distinct disorders, but two extremes of the same medical problem, having sometimes identical mutations in gene $A B C B 11$ [3]. Theoretically, whole spectrum of phenotypes between BRIC and PFIC may occur in an individual patient with mutated bile salt export pump (BSEP). According to current knowledge PFIC2 is confined to the liver, at times associated with biliary stone disease [4].

We present an unusual case of delayed clinical expression of PFIC2 presenting as recurrent intrahepatic jaundice with coexisting cholelithiasis, Gilbert's syndrome and chronic calcifying pancreatitis.

\section{CASE REPORT}

In April 2007 a 20-year-old male presented in our department with a 4-week history of spontaneously resolving jaundice (maximum bilirubin level was $22 \mathrm{mg} / \mathrm{dl}$ ). The patient denied alcohol or intravenous drug use, and had no history of allergy to medication. He reported tan stools, moderate fatigue and mild pruritus with no abdominal pain or fever. He was well developed and well nourished.

The anamnesis disclosed numerous, very similar icteric episodes since age 12 . His older sister was healthy, and the

*Address correspondence to this author at the Department of Gastroenterology, Medical University of Silesia, Katowice, Poland;

E-mail: lakmus@poczta.wprost.pl
Gilbert's syndrome was diagnosed in his older brother. The liver biopsy performed in 1999 revealed giant-cell hepatitis with significant intracellular cholestasis and minimal fibrosis (not presented). Annual frequency of icteric episodes ranged from two to four. They were accompanied by moderate weight loss and elevated serum levels of conjugated bilirubin and bile acids. Serum levels of $\gamma$-glutamyltransferase (GGT) were consistently normal. The first icteric episode was preceded by a flu-like infection, but others were not linked to identifiable factors. Abdominal discomfort or nausea was sometimes observed during icteric episodes, but fever, leukocytosis or elevated serum level of CRP have never been found. The patient was treated with ursodeoxycholic acid, which neither prevented an icteric episode, nor had a clear effect on its duration.

One year after the first icteric episode the patient started to complain of epigastric pain. Serum amylase level was normal but urinary amylase was icreased to $1600 \mathrm{U} / 1$. Abdominal ultrasound and computerized tomography (CT) revealed calcifying pancreatitis and gallbladder stones. Biliary and pancreatic anatomical malformations and obstructive choledochal disease were ruled out by endoscopical retrograde (ERCP) and magnetic resonance (MRCP) cholangiopancreato-graphy. Serum lipid profile and serum calcium level $(2.45-2.74 \mathrm{mmol} / \mathrm{l})$ were within normal ranges. In November 2002 a cholecystectomy with concurrent Rouxen-Y hepaticojejunostomy was performed. In January 2006 the patient underwent endoscopical sphincterotomy of minor papilla with removal of numerous calculi from pancreatic duct. The liver biopsy performed a few months later revealed intracellular cholestasis and marked fibrosis (Fig. 1).

Genetic studies detected no mutations in genes CFTR, SPINK1 and PRSS1, responsible for hereditary pancreatitis. Genotyping of the UGT1A1 gene promoter revealed homozygous haplotype [-3279G; A(TA)7TAA] compatible with Gilbert's syndrome. 
Two novel heterozygous mutations, c.23G $>\mathrm{C}$ (pArg8Pro) and c.3214-3C $>\mathrm{G}$ were found in $A B C B 11$ gene by sequence
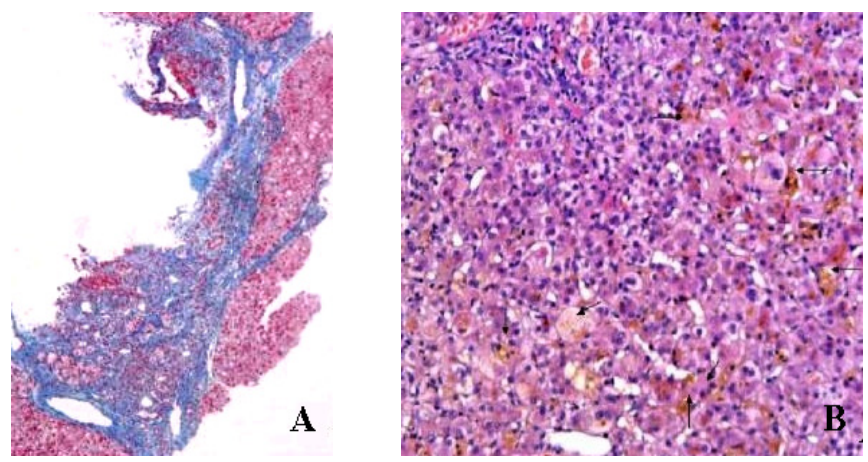

Fig. (1). Liver histopathology: (A) Severe portal and periportal fibrosis with parenchymal nodularity and paucity of biliary ducts (Azan stain, 50x). (B) Hepato-cellular bile stasis (arrows; H\&E, 200x).

analysis. Presence of both mutations was confirmed by PCR$M n / I R F L P$ and PCR-TaqIRFLP, respectively. While the mutation c. $23 \mathrm{G}>\mathrm{C}$ has been found neither in the patient's mother, nor in his siblings, mutation c. $3214-3 \mathrm{C}>\mathrm{G}$ was found in both mother and brother (Fig. 3). Neither of such mutations had been found in 30 control chromosomes. Both mutations are likely pathogenic. Arginine at position 8 is highly conserved (Fig. 3), and its substitution with proline leads to loss of positive charge in the protein chain. Mutation c.3214-3C $>\mathrm{G}$ likely generates an alternative acceptor splice
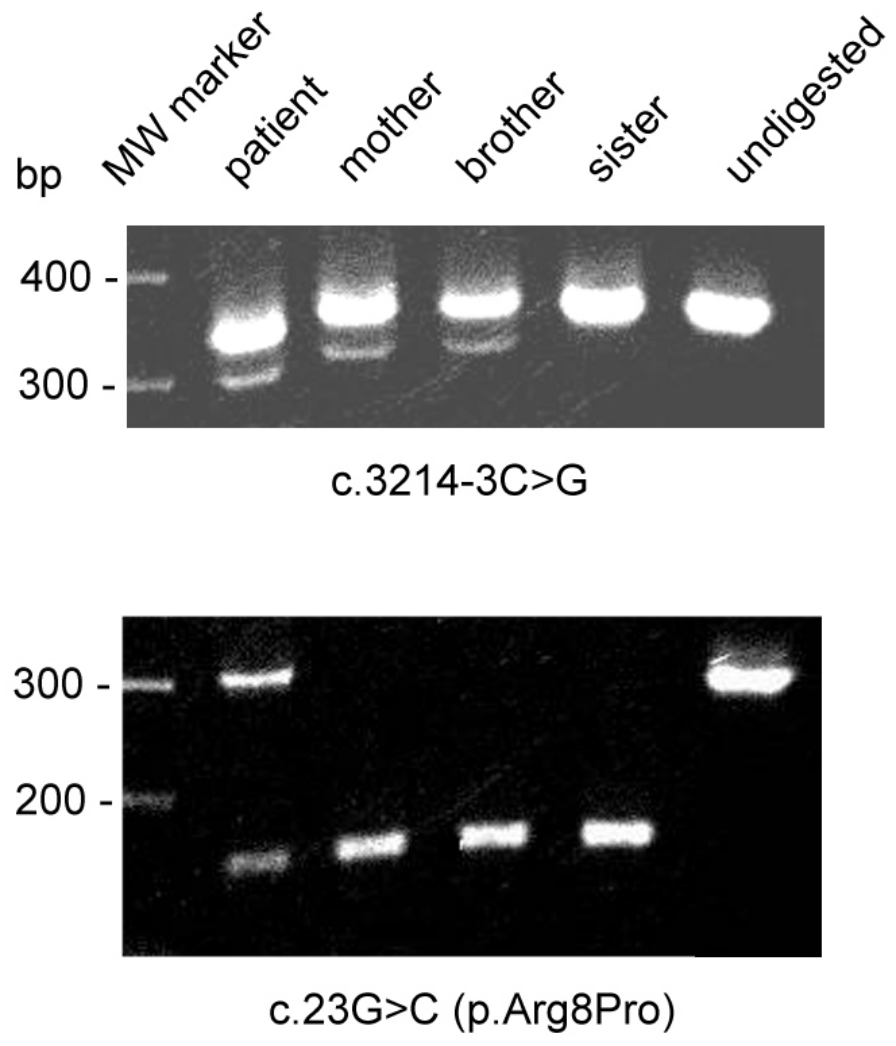

site $2 \mathrm{bp}$ upstream of the normal acceptor splice site of exon 25. Aberrrant splicing (Fig. 2) leads to frameshift and protein truncation at codon no. 1097. There was no abnormality in the coding sequence and adjacent intronic regions of ATP8B1 (PFIC1).

On follow-up presentation (May 2007) serum bilirubin level was $4.0 \mathrm{mg} / \mathrm{dl}$ (indirect bilirubin was $3.26 \mathrm{mg} / \mathrm{dl}$ ). Serum level of alanine aminotransferase was $48 / \mathrm{U} / 1$, aspartate aminotransferase was $46 \mathrm{U} / 1$ and alkaline phosphatase was $105 \mathrm{U} / \mathrm{l}$. The albumin level, prothrombin index and international normalized ratio were $4.1 \mathrm{~g} / \mathrm{dl}, 74 \%$ and 1.25 , respectively. Serum level of GGT was normal. Other laboratory studies demonstrated thrombocytopenia $\left(117.000 / \mathrm{mm}^{3}\right)$, negative hepatitis $\mathrm{B}$ and $\mathrm{C}$ serological markers, and negative autoimmune hepatitis serology. An upper endoscopy revealed small varices in the esophagus and abdominal ultrasound revealed considerably enlarged spleen (longitudinal size was $18 \mathrm{~cm})$ with dilated portal vein $(\varphi 18 \mathrm{~mm})$. CT showed in the pancreas diffuse parenchymal calcifications and dilated pancreatic duct (Fig. 3).

\section{DISCUSSION}

The reported case is of considerable clinical interest for at least two reasons i.e., unusual clinical course and development of chronic calcifying pancreatitis.

Recent clinical and genetic experience has shown that BRIC and PFIC lie at opposing ends of spectrum severity of the same disease. There is also evidence that patients with $A B C B 11$ mutations may present a wide range of phenotypes,

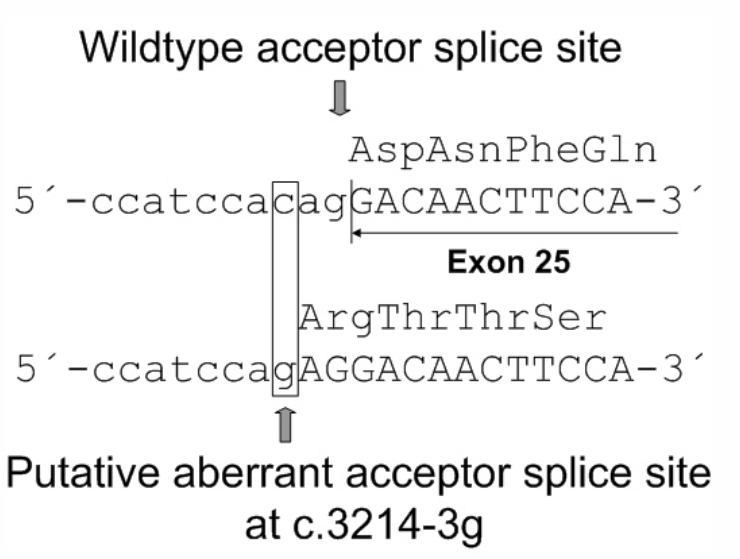

Homo sapiens

Oryctolaguc cuniculus

Mus musculus

Rattus norvegicus

Raja erinacea

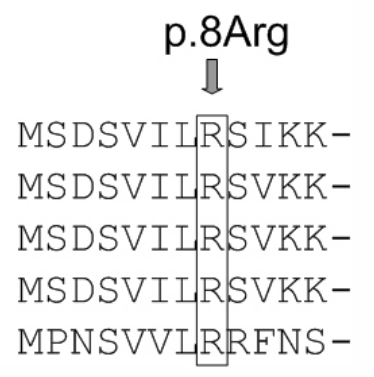

Conservation of $\mathrm{p} .8 \mathrm{Arg}$ in $\mathrm{ABCB} 11$ protein

Fig. (2). Mutations in $A B C B 11$ found in reported patient and his family members. Both mutations were detected as PCR-RFLP (left panels). Mutation c.3214-3C $>$ G likely affects splicing as depicted on the upper right panel. The lower right panel documents conservation of arginine at the aminoacid position 8 . 
from severe fibrosis to complete spontaneous resolution of liver injury or even no clinical disease at all $[4,5]$.

Our patient presented a rare intergrade phenotype linking clinical features of BRIC and PFIC. The initial clinical presentation strongly suggested BRIC in view of recurrent icteric episodes associated with subtle histopatholological lesions in the liver. Episodic character of liver disease has not changed, but check-up liver biopsy performed seven years after initial presentation showed a significant worsening of fibrosis that was no more compatible with the diagnosis of BRIC. Development of splenomegaly and small esophageal varices was keeping up with marked fibrosis found at liver biopsy.

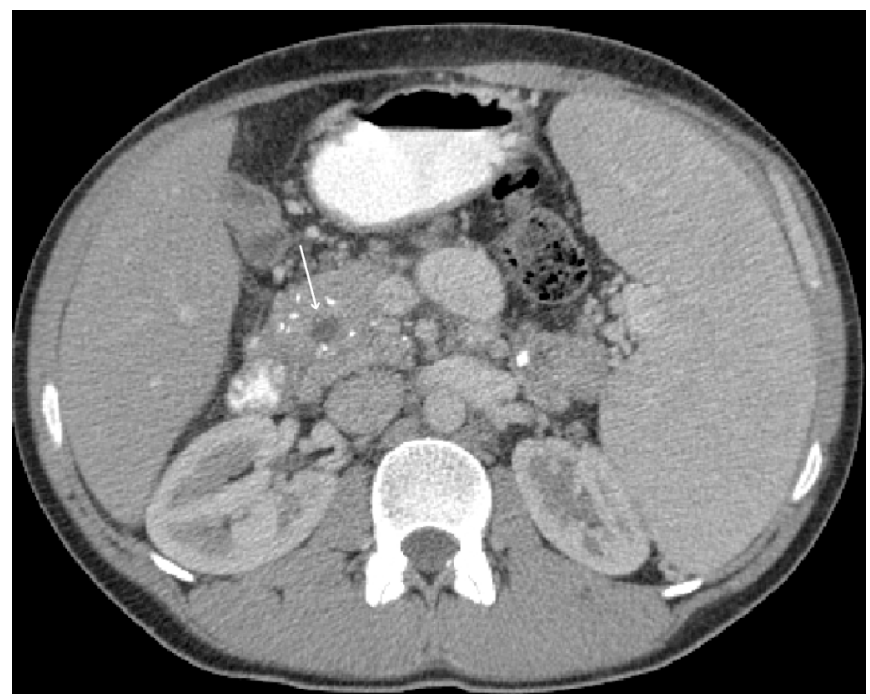

Fig. (3). Abdominal CT shows an enlarged head of pancreas with parenchymal calcifications and dilated pancreatic duct (arrow).

The clinical course of liver disease in our patient was not modified by additional factors as we did not find metabolic, autoimmune or viral etiology of liver disease. Hepaticojejunostomy might have a deleterious effect on the liver, due to potential refluxes of bowel contents to the biliary tract. However, concerning afebrile course of disease and absence of leukocytosis this concept should be rather rejected.

Mutations in $A B C B 11$ gene are deemed to have exclusively hepato-biliary consequences. It is known that BSEP deficiency may be associated with cholelithiasis, as the ratio of biliary cholesterol to bile salts is considerably increased in this condition [6]. In our patient the gallbladder stones were found with no evidence of choledochal obstructive disease on laboratory (normal GGT) and imaging (MRCP, ERCP) work-up. It is the first report on coexistence of PFIC2 with chronic calcifying pancreatitis. The calcifying pancreatitis is rather unlikely outcome of isolated cholelithiasis. While gallstones or biliary sludge are known to induce acute biliary pancreatitis, the link between recurrent acute pancreatitis and chronic pancreatitis is not complete and early cholecystectomy prevents further biliary-pancreatic interactions. Metabolic factors such as hyperlipidemia or hypercalcemia were not responsible for chronic pancreatitis, either. Moreover, we ruled out biliary and pancreatic anatomic malformations. Most common cause of calcifying pancreatitis in children is hereditary pancreatitis, which can be related to genetic mutations in the PRSS1 (encodes cationc tripsinogen), Kazal type 1 (SPINK1) or the cystic fibrosis transmembrane conductance regulator (CFTR). In the reported patient the DNA sequencing did not show any of these mutations, however, it should be remembered that in minority of cases still unknown mutations may be responsible for idiopathic pancreatitis.

Taking into account almost simultaneous emergence of chronic pancreatitis and PFIC2, it would be tempting to postulate common pathophysiology between these diseases. Chronic pancreatitis has been occasionally recognized in patients with $A T P 8 B 1$ mutations, although the role of FIC1 protein in production of pancreatic juice remains to be elusive [7]. It could be hypothesized that only certain $A B C B 11$ mutations and/or prolonged survival could contribute to pancreatic injury in PFIC2 patients. However, due to very limited knowledge on extrahepatic expression and function of BSEP, no conclusion about etiology of calcifying pancreatitis can be made at this point.

In conclusion, we have presented a diagnostically challenging patient presenting slowly developing PFIC2 with concurrent cholelithiasis, Gilbert's syndrome and chronic calcifying pancreatitis.

\section{REFERENCES}

[1] Scheimann AO, Strautnieks SS, Knisely AS, Byrne JA, Thompson RJ, Finegold MJ. Mutations in bile salt export pump (ABCB11) in two children with progressive familial intrahepatic cholestasis and cholangiocarcinoma. J Pediatr 2007; 150 (5): 556-9.

[2] Knisely AS, Strautnieks SS, Meier Y, et al. Hepatocellular carcinoma in ten children under five years of age with bile salt export pump deficiency. Hepatology 2006; 44 (2): 478-86.

[3] Van Mil SW, van der Woerd WL, van der Brugge G, et al. Benign recurrent intrahepatic cholestasis type 2 is caused by mutations in ABCB11. Gastroenterology 2004; 127 (2): 379-84.

[4] Van Mil SW, Houwen RH, Klomp LW. Genetics of familial intrahepatic cholestasis syndromes. J Med Genet 2005; 42 (6): 449-63.

[5] Wang L, Soroka CJ, Boyer JL. The role of bile salt export pump mutations in progressive familial intrahepatic cholestasis type II. J Clin Invest 2002; 110 (7): 965-72.

[6] Kong FM, Sui CY, Li YJ, Guo KJ, Guo RX. Hepatobiliary membrane transporters involving in the formation of cholesterol calculus. Hepatobiliary Pancreat Dis Int 2006; 5 (2): 286-9.

[7] Walkowiak J, Jankowska I, Pawlowska J, Bull L, Herzig KH, Socha J. Normal pancreatic secretion in children with progressive familial intrahepatic cholestasis type 1. Scand J Gastroenterol 2006; 41 (12): 1480-3. 